\title{
Magnesium hydride for energy storage applications: The kinetics of dehydrogenation under different working conditions
}

Antonio Perejón ${ }^{\mathrm{a}, \mathrm{b}, *}$, Pedro E. Sánchez-Jiménez ${ }^{a}$, José M. Criado ${ }^{a}$, Luis A. PérezMaqueda $^{a, *}$

${ }^{a}$ Instituto de Ciencia de Materiales de Sevilla (C.S.I.C.-Univ. Sevilla). C. Américo Vespucio 49, Sevilla 41092. Spain

${ }^{b}$ Departamento de Química Inorgánica, Facultad de Química, Universidad de Sevilla, Sevilla 41071, Spain

\begin{abstract}
A new approach to the kinetics of magnesium hydride dehydrogenation is considered. A model able to predict the dehydrogenation under different experimental conditions has been proposed. A new combined kinetic analysis method, which considers the thermodynamic of the process according to the microreversibility principle, has been used for performing the kinetic analysis of data obtained under different thermal schedules at hydrogen pressures ranging from high vacuum up to 20 bar.

The kinetic analysis shows that the dehydrogenation mechanism of magnesium hydride depends on the experimental conditions. Thus, the reaction follows a first order kinetics, equivalent to an Avarmi-Erofeev kinetic model with an Avrami coefficient equal to 1, when carried out under high vacuum, while a mechanism of tridimensional growth of nuclei previously formed (A3) is followed under hydrogen pressure. An explanation of the change of mechanism is given. It has been shown that the activation energy is closed to the $\mathrm{Mg}-\mathrm{H}$ bond breaking energy independently of the hydrogen pressure surrounding the sample, which suggests that the breaking of this bond would be the rate limiting step of the process. The reliability of the calculated kinetic parameters is tested by comparing simulated and experimental curves.
\end{abstract}

Keywords: magnesium hydride; hydrogen storage; thermal energy storage; kinetic analysis; dehydrogenation-hydrogenation reactions; kinetic model 


\section{Introduction}

Magnesium hydride is a material of the most interest for a number of technical applications, mainly as hydrogen storage material for PEM fuel cells, due to its large reversible storage capacity (7.6 mass $\%$ ) of high purity hydrogen [1-5], and as a thermal energy storage system in thermosolar plants due to the high enthalpy of the hydrogenation-dehydrogenation reactions [6-10]. Moreover, magnesium has a relatively high abundance in earth. For any of these two applications, the kinetics of the dehydrogenation-hydrogenation reactions is of paramount importance. It has been reported in literature that the dehydrogenation reaction is a very sensitive process that depends on a number of parameters such as thermal history of the sample (for example, activation cycles of hydrogenation-dehydrogenation) [4], structural defects in the sample produced by mechanical milling [11], presence of additives with or without catalytic effect [12-16], gas pressure [17-18], etc. Moreover it has been shown in literature that the influence of the transport of hydrogen through the $\mathrm{Mg} / \mathrm{MgH}_{2}$ bed on the kinetics of hydrogenation/dehydrogenation reactions must be considered when either large or compacted samples are being processed [18-20]. This is not the case of this work, in which very small amounts of powder samples will be used for minimizing the influence of gas transport phenomena on the forward reaction.

A deep knowledge of the kinetics of absorption and desorption processes in noncatalyzed $\mathrm{MgH}_{2}$-based materials is crucial for future applications both as heat storage or hydrogen storage material and is of the most interest to better understand the behavior of milled and catalyzed $\mathrm{MgH}_{2}$.

A very large number of studies of absorption and desorption of hydrogen in $\mathrm{MgH}_{2}$ and related compounds have been performed employing either volumetric analysis under isothermal conditions or thermogravimetry (TG) and differential scanning calorimetry (DSC) at linear rising temperature under a flow of hydrogen or an inert gas [11, 17-18, 21-28]. However, in most of the cases the hydrogen pressure has not been carefully controlled and, provided that the dehydrogenation of $\mathrm{MgH}_{2}$ is reversible, the uncontrolled gradients of hydrogen pressure generated during the reaction could lead to unrealistic kinetic models and false kinetic parameters. Moreover, in many cases the kinetic analysis has been carried out by previously assuming a given kinetic model for fitting the experimental data, but it is known, firstly, that a same set of experimental data can be simultaneously fitted by a number of kinetic models and, secondly, that the 
activation energy is strongly dependent on the kinetic model previously assumed [2933]. A proper kinetic analysis of reversible thermal decomposition reactions under a given constant pressure of the gas self-generated in the reaction would imply to consider the thermodynamic of the process by taking into account the microreversibility principle $[26,30,34-35]$. On the other hand, it would be advisable to associate the microreversibility principle with methods of kinetic analysis that would allow determining both the kinetic parameters and the kinetic model obeyed by the reaction without any previous assumption on the kinetic model.

The objective of this work is the study of the dehydrogenation kinetics of magnesium hydride at pressures ranging from high vacuum up to 20 bars of hydrogen in order to get a unified reaction mechanism that would allow predicting the kinetic behavior of the dehydrogenation of magnesium hydride as a function of the hydrogen pressure.

\section{Experimental}

Commercially available magnesium hydride purchased from Aldrich (product number 683043 , with average particle size of $50 \mu \mathrm{m}$ ) was used for performing the study. In all cases, the powders were weighted inside a glove-box, taken out of it and immediately placed in the corresponding instrument in order to minimize the exposition of the samples to the air.

Thermogravimetric measurements were performed using a high sensitivity $\left(2 \times 10^{-7} \mathrm{~g}\right)$ CI Electronics thermobalance and a fast response furnace connected to a high-vacuum system (rotary and turbomolecular pumps) that is able to reduce pressure to $\sim 5 \times 10^{-5}$ mbar. A set of calibration weights where used to calibrate the mass output of the themobalance, while the temperature calibration was performed using hydrous calcium oxalate heated at $10 \mathrm{~K} \mathrm{~min}^{-1}$. The experiments were performed at $\sim 5 \times 10^{-5} \mathrm{mbar}$ in conventional linear heating rate conditions, at $0.2 \mathrm{~K} \mathrm{~min}^{-1}, 0.5 \mathrm{~K} \mathrm{~min}^{-1}$ and $1 \mathrm{~K} \mathrm{~min}^{-1}$. The steady-state of the system was reached after outgassing overnight at room temperature and full pumping rate. A quadrupole mass spectrometer (Prism, Pfeiffer) was employed to perform the evolved gas analysis. A shutoff valve allows carrying out the experiments either under high vacuum or in a gas flow. Typical sample size was $\sim 85$ $\mathrm{mg}$, which was placed in alumina pan.

Differential scanning calorimetry (DSC) experiments were performed under 10 bar and 20 bar of hydrogen pressure on a pressure DSC system (Q20P, TA Instruments, 
Crawley, UK) that provides heat flow measurements on pressure sensitive materials. The pressure cell employs standard heat flux DSC technology and incorporates pressure control valves, a pressure gauge, and over-pressure protection. The system was connected to a mass flow controller and a pressure controller in order to carry out the experiments under $50 \mathrm{~cm}^{3} \mathrm{~min}^{-1}$ hydrogen flow and at constant pressure. The heat flow and temperature calibration were carried out employing standard sapphire discs and indium. The metal was heated through its melting transition and the calculated heat of fusion compared to the theoretical value. Moreover, the recorded melting point of this standard is compared to the known melting point and the difference is calculated for temperature calibration. The DSC experiments were carried out in open alumina pans at different heating rates, $\beta: 1 \mathrm{~K} \mathrm{~min}^{-1}, 2.5 \mathrm{~K} \mathrm{~min}^{-1}, 5 \mathrm{~K} \mathrm{~min}^{-1}$ and $7.5 \mathrm{~K} \mathrm{~min}^{-1}$. Typical sample size was $\sim 5 \mathrm{mg}$. Before performing the experiments, the system was outgassed using a rotary valve during $20 \mathrm{~min}$, to prevent sample oxidation. The experimental DSC curves were normalized in such a way that the total area enclosed corresponds to full conversion (i.e; $\alpha=1$ ). Thus, the values of $d \alpha / d T$ at a given temperature are equal to the value of the ordinate (y axis) at this temperature and the corresponding value of $d \alpha / d t$ is obtained through the expression $\mathrm{d} \alpha / \mathrm{dt}=\beta(\mathrm{d} \alpha / \mathrm{dT})$. The $\alpha$-T plots were obtained by numerical integration of the normalized DSC plots.

X-ray diffraction patterns were recorded in vacuum in a Philips X'Pert Pro diffractometer working at $45 \mathrm{kV}$ and $40 \mathrm{~mA}$, using $\mathrm{CuK} \alpha$ radiation and equipped with an $X^{\prime}$ Celerator detector and a graphite diffracted beam monochromator.

\section{Theoretical}

The dehydrogenation of magnesium hydride takes place according to the following reversible reaction:

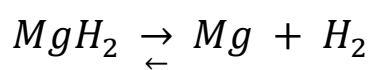

According to the microreversibility principle, in a reversible reaction the mechanism in one direction is exactly the reverse of the mechanism in the other direction. This principle has been employed by different authors to study the influence of product gas 
pressure on reversible reactions, leading to the following expression for the reaction rate $[26,30,34-37]$ :

$$
\frac{d \alpha}{d t}=A e^{-\frac{E}{R T}} f(\alpha)\left(1-\frac{p}{p^{*}}\right)
$$

where $\alpha$ is the reacted fraction calculated normalizing the mass loss in thermogravimetry or integrating and normalizing the DSC curves, $A$ is the preexponential factor, $\mathrm{E}$ the activation energy, $\mathrm{f}(\alpha)$ the kinetic model followed by the reaction, $p$ the pressure of the gas generated in the reaction (which is maintained constant in our experiments) and $p^{*}$ is the equilibrium pressure of the gas (hydrogen in our case). If the reaction is carried out under high vacuum in such a way that the pressure $p$ is extremely low with regard to the equilibrium pressure, the term $p / p^{*}$ would be close to zero and equation ( $2 \mathrm{a})$ becomes:

$$
\frac{d \alpha}{d t}=A e^{-\frac{E}{R T}} f(\alpha)
$$

The equilibrium pressure in equation (2a) would be determined as a function of the temperature from the Van't Hoff equation:

$$
\ln \frac{p^{*}}{p_{0}}=\frac{\Delta S}{R}-\frac{\Delta H}{R T}
$$

where $\mathrm{p}^{*}$ is the equilibrium pressure at the absolute temperature $\mathrm{T}, \mathrm{p}_{0}$ is the atmospheric pressure, $\Delta \mathrm{H}$ and $\Delta \mathrm{S}$ are the enthalpy and entropy of hydride formation and $\mathrm{R}$ is the universal gas constant.

\subsection{Model free methods}

Friedman isoconversional method allows determining the activation energy as a function of $\alpha$ without considering the kinetic model of the process [38-39]. Thus, equation (2a) can be rearranged in logarithmic form as follows: 


$$
\ln \left(\frac{d \alpha}{d t}\right)-\ln \left(1-\frac{p}{p *}\right)=\ln [A f(\alpha)]-\frac{E}{R T}
$$

If we are far from equilibrium like in the experiments conducted under high vacuum we get from equation $(2 b)$ :

$$
\ln \left(\frac{d \alpha}{d t}\right)=\ln [A f(\alpha)]-\frac{E}{R T}
$$

The activation energy at a constant $\alpha$ is calculated from the slope of the plot of the lefthand side of either equation (4a) or (4b) against the inverse of temperature at a given value of $\alpha$.

\subsection{Combined kinetic analysis}

The combined kinetic analysis is a method for obtaining both the kinetic model and the kinetic parameters associated to a process from the simultaneous analysis of a set of data obtained under different thermal schedules without previously assuming neither of the kinetic parameters or the kinetic model of the process [40-41]. The modified SestakBerggren equation, i.e. $f(\alpha)=c(1-\alpha)^{n} \alpha^{m}$, has been considered for this purpose. This is because this empirical equation behaves like an umbrella that fit all the kinetic equations proposed in literature for describing solid state reactions, including deviations from ideal models [40, 42-43]. Thus, the equation of Sestak-Berggren simplifies considerably the kinetic analysis, allowing the discrimination of the kinetic model in a second step with the help of master plots.

The equation for the combined kinetic analysis is obtained rearranging the equation (2a) in logarithmic form after replacing the $\mathrm{f}(\alpha)$ function by the Sestak-Berggren equation:

$$
\ln \left[\frac{d \alpha / d t}{(1-\alpha)^{n} \alpha^{m}\left(1-\frac{p}{p^{*}}\right)}\right]=\ln (c A)-\frac{E}{R T}
$$

or

$$
\ln \left[\frac{d \alpha / d t}{(1-\alpha)^{n} \alpha^{m}}\right]=\ln (c A)-\frac{E}{R T}
$$


when far from equilibrium (i.e.; $p / p^{*}=0$ ). The set of experimental data, corresponding to different heating schedules, is substituted either into equation (5a) or (5b) and the left-hand side of the equations is plotted versus the inverse of temperature. The Pearson linear correlation coefficient is set as an objective function for optimization, and the values of the parameters $\mathrm{n}$ and $\mathrm{m}$ that provide the best linear fit to the plot are determined. The values of $\mathrm{E}$ and $\ln (\mathrm{cA})$ are calculated from the slope and intercept respectively.

\section{Results and discussion}

\subsection{Kinetics of magnesium hydride dehydrogenation in vacuum.}

Figure 1 presents the XRD pattern of the as received magnesium hydride recorded in vacuum at room temperature in the $2 \theta$ range from $25^{\circ}$ to $50^{\circ}$. The Rietveld refinement of the XRD profile shows that the sample is constituted by a mixture of $86 \% \mathrm{of} \mathrm{MgH}_{2}$ and $14 \%$ of metallic magnesium. The lattice parameters determined from the Rietveld method are $\mathrm{a}=0.4518(4)$ and $\mathrm{c}=0.3019(3)$ that are in excellent agreement with the values reported for the tetragonal $\beta-\mathrm{MgH}_{2}$ phase according to the powder diffraction file JCPDS 12-0697. These results suggest that the sample is constituted by quasistoichiometric $\mathrm{MgH}_{2}$ and unreacted magnesium. It is noteworthy to point out that it has been theoretically demonstrated in literature that the $\beta-\mathrm{MgH}_{2}$ phase is capable of accommodating only very small concentrations of hydrogen vacancies that are mainly isolated rather than forming clusters [44]. It has been suggested that the formation of non-stoichiometric magnesium hydride is restricted to nanometric particles, although structural evidence has not been reported until now [3, 22, 45]. On the other hand, it is known that, for bulk micron sized particles a shell of magnesium hydride is formed, which prevents the hydrogenation of the remaining metal core after the maximum hydrogenation of magnesium is achieved [3, 45-47]. Thus, it can be considered that the percentage of magnesium present in the sample is not coming from the partial decomposition of $\mathrm{MgH}_{2}$ during the storage under inert atmosphere but it corresponds to unreacted metal. Therefore, it is reasonable to perform the kinetic analysis in the overall reacted fraction range, considering that $\alpha=0$ for the starting sample and $\alpha=1$ for the completely dehydrogenated material. 


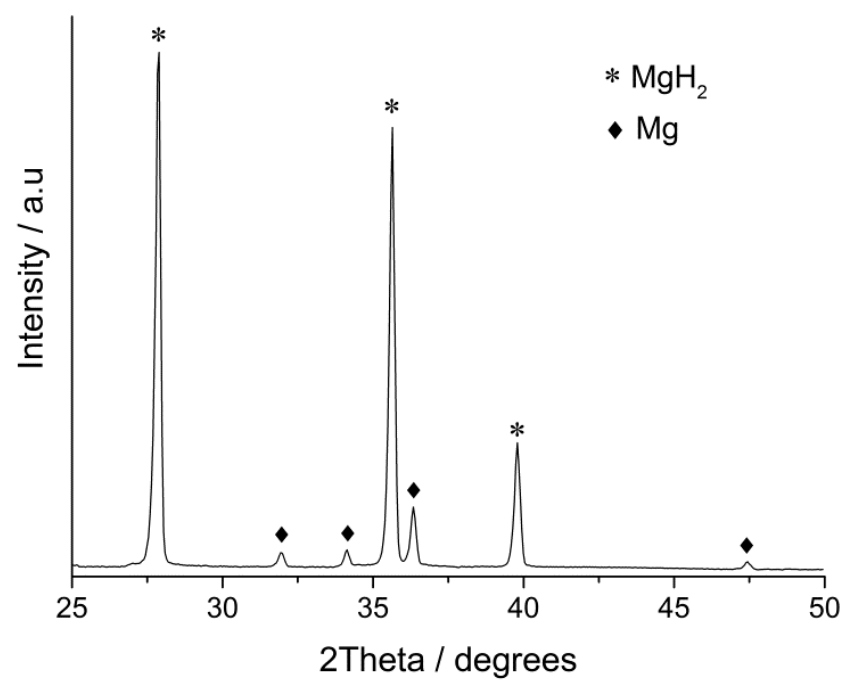

Figure 1. XRD pattern of the as received magnesium hydride recorded in vacuum at room temperature.

The kinetics of $\mathrm{MgH}_{2}$ dehydrogenation was first studied by thermogravimetry under high vacuum to prevent oxidation at the time to approach to zero the ratio $p / p^{*}$ in order to obtain directly the activation energy of the forward reaction (1) from equation (4b) and to perform the combined kinetic analysis by means of equation (5b). Experiments were performed at low heating rates with a double purpose: firstly, to reduce the decomposition temperatures in order to avoid $\mathrm{Mg}$ sublimation and secondly, to minimize the influence of heat and mass transfer phenomena on the forward reaction.

Figure 2 shows the mass loss and the rate of mass loss profiles for the thermal dehydrogenation of magnesium hydride recorded under $\sim 5 \times 10^{-5}$ mbar at $0.2 \mathrm{~K} \mathrm{~min}^{-1}$, and the corresponding evolution of the $\mathrm{H}_{2}$ signals registered by mass spectrometry as a function of temperature. It is clear from Figure $2 \mathrm{a}$ that hydrogen is released in a single process and the mass loss is $6.5 \%$, what indicates that the starting sample is constituted by $86 \%$ of $\mathrm{MgH}_{2}$, which confirms the result previously obtained from the XRD analysis by the Rietveld method. Figure $2 b$ confirms that $\mathrm{H}_{2}$ is evolved in a single stage, as observed in the thermogravimetric trace. 

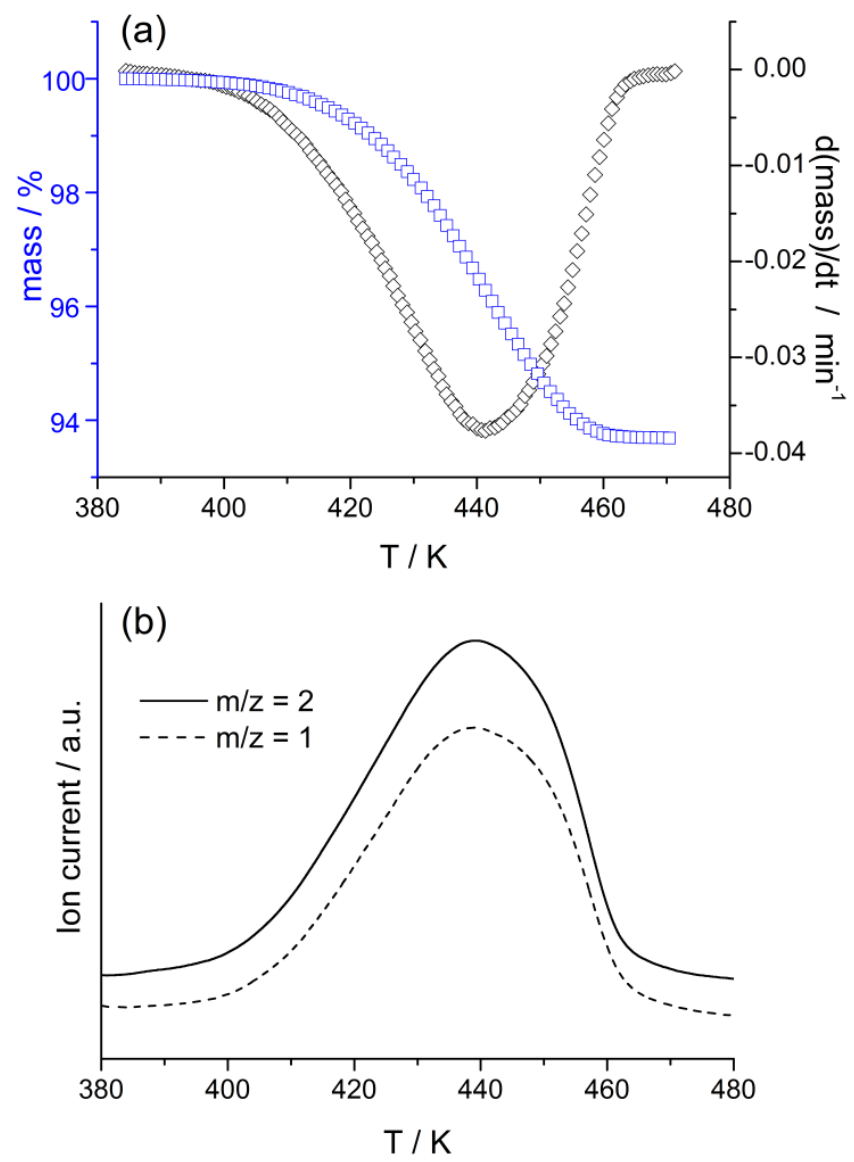

Figure 2. (a) Integral TG (mass loss) and differential TG curves for the thermal dehydrogenation of magnesium hydride recorded under $\sim 5 \times 10^{-5}$ mbar at $0.2 \mathrm{~K} \mathrm{~min}^{-1}$. (b) Corresponding $\mathrm{H}_{2}$ signals registered by mass spectrometry as a function of temperature.

Figure 3 presents the thermogravimetric curves recorded at $0.2 \mathrm{~K} \mathrm{~min}^{-1}, 0.5 \mathrm{~K} \mathrm{~min}^{-1}$ and $1 \mathrm{~K} \mathrm{~min}^{-1}$. Mass losses have been normalized to turn it into reacted fractions. Nevertheless, an overall mass loss equal to $6.5 \%$ was obtained in the whole set of experiments shown in Figure 3. The shape of the $\alpha$-T plots included in this figure supports that the dehydrogenation of $\mathrm{MgH}_{2}$ occurs through a single step. The fact that some authors have observed more than one step perhaps would be explained considering the contamination of their samples with $\mathrm{Mg}(\mathrm{OH})_{2}$ coming from a certain hydrolysis of the hydride [17, 21]. Thus, the thermal decomposition of $\mathrm{MgH}_{2}$ and $\mathrm{Mg}(\mathrm{OH})_{2}$ could be overlapping.

The apparent activation energy of $\mathrm{MgH}_{2}$ dehydrogenation as a function of the reacted fraction was calculated by means of the Friedman isoconversional method described in section 3.1, analyzing simultaneously all experimental curves included in Figure 3 at different $\alpha$ values by means of equation (4b), provided that $p / p *$ is close to zero. In fact, 
the values of $p^{*}$ in the range of temperatures at which the dehydrogenations of $\mathrm{MgH}_{2}$ are recorded in Figure 3 are higher than 30 mbar according with the equilibrium data reported by Zhou et al. [48-49], what means that the values of $p / p^{*}$ are lower than $10^{-6}$ all over the decomposition range.

Table 1 presents the evolution of the apparent activation energy with conversion, which remains approximately constant during the whole process, averaging $111 \pm 7 \mathrm{~kJ} \mathrm{~mol}^{-1}$. These results support that magnesium hydride dehydrogenation would take place through a single step that can be described by a unique kinetic triplet. The apparent monotonous decrease of activation energy as $\alpha$ increases would be understood taking into account that the weight of the determination of this parameter from conventional Friedman method rests on the heating rate and, therefore, systematic small variations of the heating rate with the temperature would lead to monotonous variation of the activation energy. This uncertainty would be further avoided using the combined kinetic analysis methods that lead to statistically significant data because very large number of $\alpha$-T points, coming from sets of $\alpha$-T plots obtained under different heating schedules is simultaneously processed.

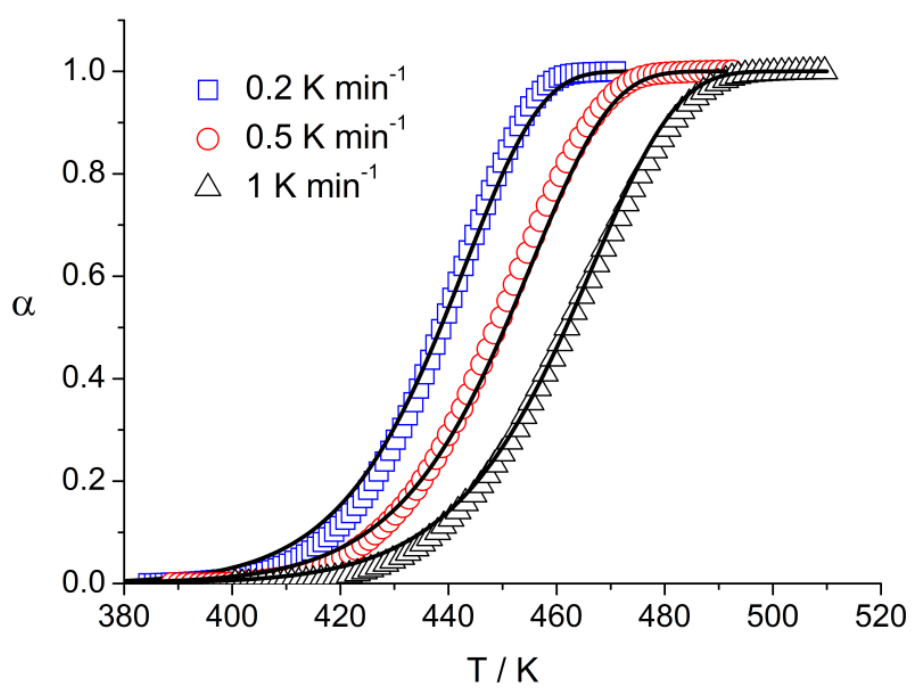

Figure 3. Experimental curves (dotted lines) corresponding to the thermal dehydrogenation of magnesium hydride under high vacuum registered at $0.2,0.5$ and $1 \mathrm{~K} \mathrm{~min}^{-1}$. The curves reconstructed using the kinetic parameters obtained from the analysis are plotted as solid lines. 
Table 1. Activation energy values as a function of $\alpha$, obtained from the Friedman isoconversional analysis of the experimental curves shown in Figure 3.

\begin{tabular}{ccc}
\hline $\boldsymbol{\alpha}$ & $\mathbf{r}$ & $\mathbf{E} / \mathbf{~ k J ~ m o l}^{-1}$ \\
\hline 0.1 & 0.994 & $120 \pm 9$ \\
0.2 & 0.996 & $118 \pm 8$ \\
0.3 & 0.996 & $117 \pm 5$ \\
0.4 & 0.997 & $112 \pm 3$ \\
0.5 & 0.997 & $109 \pm 4$ \\
0.6 & 0.997 & $109 \pm 4$ \\
0.7 & 0.995 & $107 \pm 6$ \\
0.8 & 0.994 & $104 \pm 8$ \\
0.9 & 0.993 & $100 \pm 10$ \\
\multicolumn{3}{c}{ Mean Ea value: $111 \pm 7 \mathrm{~kJ} \mathrm{~mol}^{-1}$} \\
\hline
\end{tabular}

In order to determine the kinetic model and the pre-exponential factor, the experimental curves in Figure 3 were also analyzed using the combined kinetic analysis methodology, described in Section 3.2. Figure 4a includes the plot of the left-had side of equation (5b) versus the inverse of temperature. The optimization procedure shows that experimental curves in Figure 3 are fitted simultaneously into a single straight line when $\mathrm{n}$ and $\mathrm{m}$ take the values 0.94 and 0 respectively. The slope of the plot leads to an apparent activation energy value of $109 \pm 1 \mathrm{~kJ} \mathrm{~mol}^{-1}$, in good agreement with the value obtained from the isoconversional method, and the intercept to a preexponential factor of $\mathrm{cA}=1.1( \pm 0.2)$ $\times 10^{11} \mathrm{~min}^{-1}$. The $\mathrm{f}(\alpha)$ function deduced from the analysis, $f(\alpha)=(1-\alpha)^{0.94}$, suggests that the process is driven by a first-order (F1) kinetic function. This result is demonstrated by the comparison with the master plots reported in literature for the different kinetic models proposed for describing solid state reactions (Figure 4b) [50]. The functions are normalized at $\mathrm{f}(0.5)$ to better distinguish the different plots. It is clear that the calculated $\mathrm{f}(\alpha)$ function and the theoretical curve corresponding to first-order kinetics match closely. It must be emphasized that the thermal decomposition of $\mathrm{MgH}_{2}$ is a heterogeneous reaction and, therefore, the term (1- $\alpha)$ cannot be interpreted like a direct representation of the concentration of the unreacted fraction in the kinetic equation. The finding of a F1 kinetic model would be explained by assuming mechanisms that imply the formation and growth of nuclei described by an Avrami-Erofeev kinetic model with an Avrami coefficient equal to 1 (A1), what leads to a kinetic equation formally identical to the F1 kinetic equation [51-54]. Other alternative explanation would be 
feasible if we take into account that it has been shown in a recent review that all the $\mathrm{MgH}_{2}$ samples available in the market are not homogeneous in size but they exhibit a large log-normal particle size distribution (PSD) [3]. Thus, the F1 kinetic equation would be representative of a reaction mechanism in which the reaction rate is controlled by the advance of the interface. This is because it has been reported in literature that the reactions following this mechanism move to an apparent F1 kinetic model as far as the particle size distribution is broadened, without altering the value of the real activation energy obtained from isoconversional methods [43].
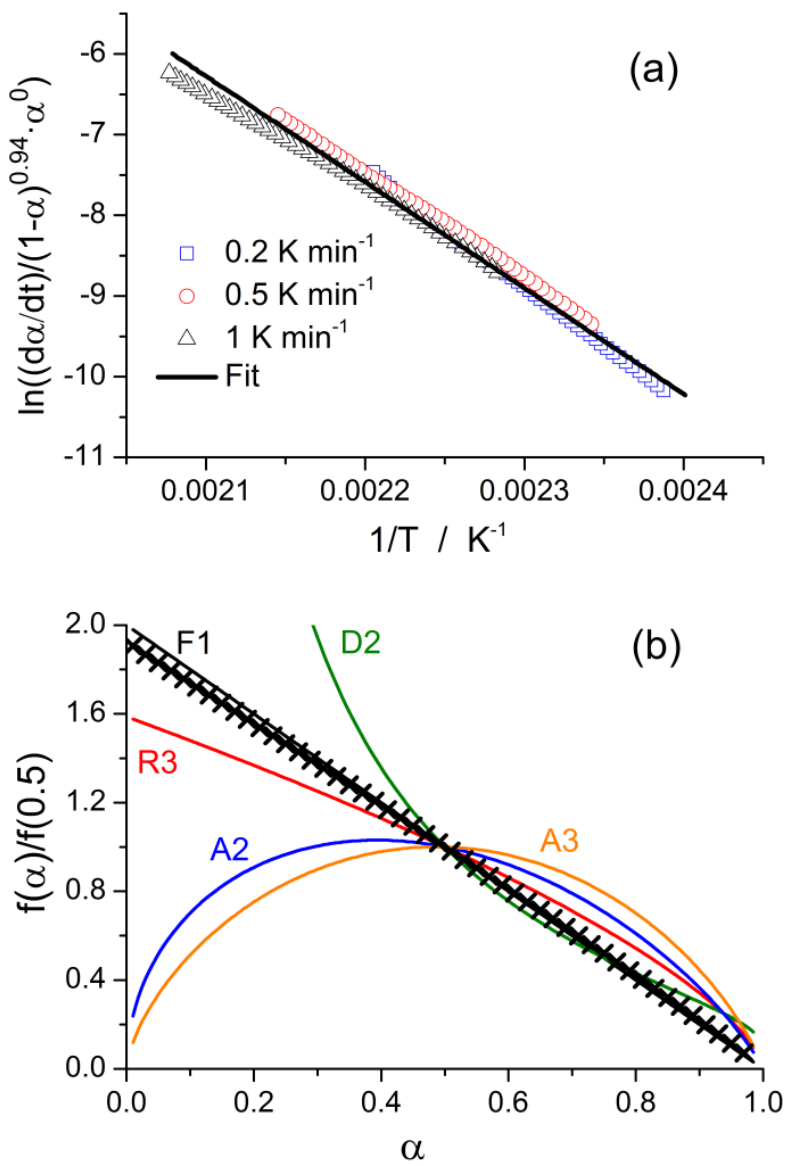

Figure 4. (a) Combined kinetic analysis plot of the experimental curves presented in Figure 3. (b) Comparison of the $\mathrm{f}(\alpha)$ functions (lines) normalized at $\alpha=0.5$ corresponding to some of the ideal kinetic models with the $\mathrm{f}(\alpha)$ function resulting from the combined analysis, $\mathrm{f}(\alpha)=(1-\alpha)^{0.94}(\mathrm{dots})$.

The original experimental curves were reconstructed by simulating a set of curves using the kinetic parameters resulting from the above analysis. The simulations were performed by integrating the general kinetic equation and considering the heating conditions used in the different experiments, and using fourth-order numerical 
integration Runge-Kutta method. It is clear in Figure 3 that simulated and experimental curves match closely, proving the validity of the kinetic parameters obtained from the combined kinetic analysis.

Figure $4 \mathrm{~b}$ shows that the comparison of the experimental master plots obtained from the simultaneous kinetic analysis of the whole set of thermogravimetric curves recorded under different heating schedules with the theoretical master plots corresponding to different kinetic models clearly allows discriminating the real kinetic model obeyed by the reaction. However, it is noteworthy emphasizing that, contrarily, the shape analysis of a single thermogravimetric curve does not allow discriminating the actual kinetic model. Thus, Fernández and Sánchez have shown that the analysis of individual $\alpha-T$ plots obtained for the dehydrogenation of $\mathrm{MgH}_{2}$ would be compatible with a first order and a diffusion kinetic model [18]. Similarly, Criado and Morales have shown, from the analysis of simulated $\alpha$-T plots, that it is impossible to discriminate between first order, diffusion and Avrami-Erofeev kinetic models from a single thermogravimetric curve obtained at a constant heating rate [55-56].

\subsection{Kinetics of magnesium hydride dehydrogenation under hydrogen pressure.}

The use of magnesium hydride for hydrogen storage or energy storage applications would necessarily take place under hydrogen pressure conditions rather than in high vacuum. Therefore, it is of the most interest to determine the effect of hydrogen pressure on magnesium hydride dehydrogenation kinetics.

The kinetics of this process was studied by DSC under 10 bar and 20 bar of constant hydrogen pressure during the entire experiment as described in the experimental section. The experimental curves were recorded under linear heating rate conditions at $1 \mathrm{~K} \mathrm{~min}^{-}$ ${ }^{1}, 2.5 \mathrm{~K} \mathrm{~min}^{-1}, 5 \mathrm{~K} \mathrm{~min}^{-1}$ and $7.5 \mathrm{~K} \mathrm{~min}^{-1}$. Figure 5a presents the as obtained DSC traces for thermal dehydrogenation of $\mathrm{MgH}_{2}$ under 10 bar of hydrogen at the four heating rates employed. It can be observed that the temperature of dehydrogenation increases more than $250 \mathrm{~K}$ with regards to the experiments performed under high vacuum. All curves show a single peak, which supports again that the dehydrogenation of $\mathrm{MgH}_{2}$ occurs through a single step as previously proposed in section 4.1. Similar curves were obtained at 20 bar although they are moved at higher temperatures as figure $5 \mathrm{~b}$ shows.

The enthalpy of $\mathrm{MgH}_{2}$ dehydrogenation can be calculated integrating the peak above the baseline. The enthalpy obtained from the DSC plots was $63.5 \mathrm{~kJ} \mathrm{~mol}^{-1}$ and 
consequently, if we consider that the percentage of $\mathrm{MgH}_{2}$ in the sample previously determined was equal to $86 \%$, it results that the enthalpy obtained for the dehydrogenation of this compound is $\Delta \mathrm{H}=73.8 \mathrm{~kJ} / \mathrm{mol}$, in good agreement with the thermodynamic data reported in literature [57-59]. This value of $\Delta \mathrm{H}$ has been used in equation (3) for calculating the equilibrium pressures as a function of the temperature demanded by equations (4a) and (5a). A value of $\Delta \mathrm{S}=130 \mathrm{~J} \mathrm{~K}^{-1} \mathrm{~mol}^{-1}$ has been taken for the entropy change associated to the forward dehydrogenation reaction according with equation (1). The value of $\Delta \mathrm{S}$ is dominated by the entropy of hydrogen gas liberated during the reaction and it is considered constant for the dehydrogenation of all the metallic hydrides $[57,59-60]$. The values of $p^{*}$ here calculated as a function of the temperature show a good agreement with those previously reported by Zhou et al. [49].
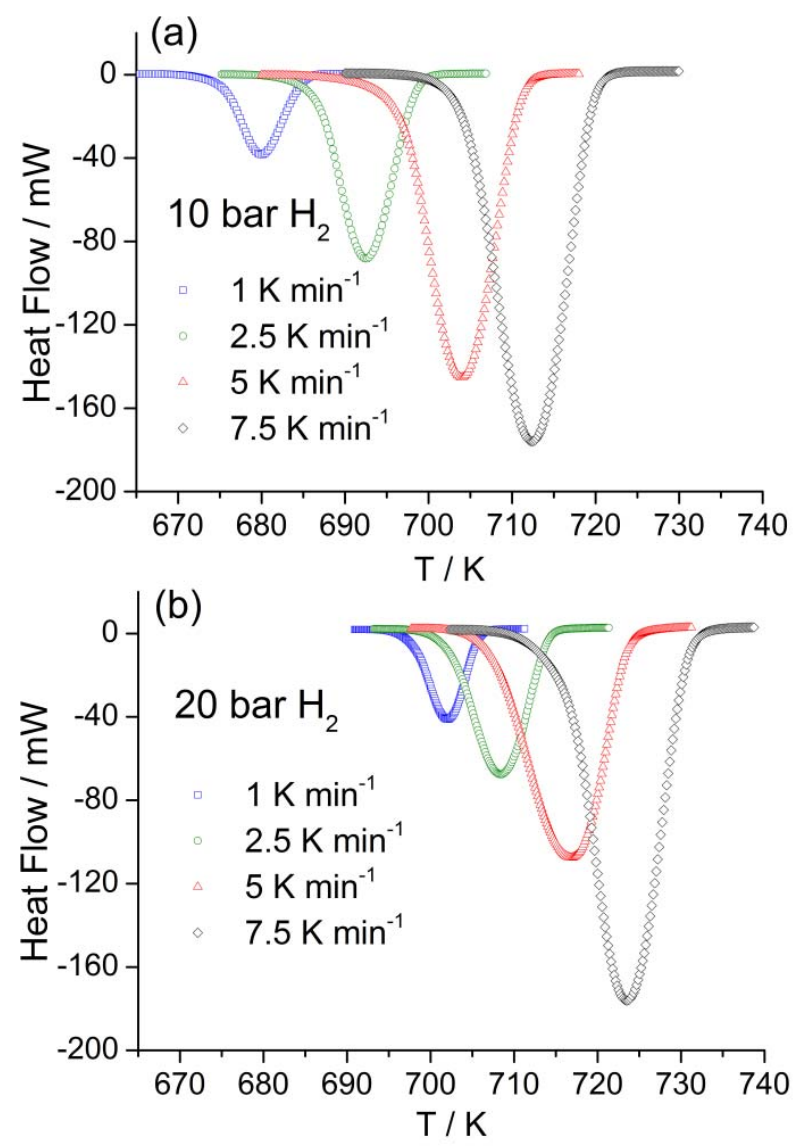

Figure 5. DSC experimental curves for thermal dehydrogenation of magnesium hydride, registered at different hydrogen pressures: a) 10 bar; b) 20 bar.

Figure 6 shows the temperature dependences of the pressure correction term $\left(1-p / p^{*}\right)$ for experiments recorded at $2.5 \mathrm{~K} \mathrm{~min}^{-1}$ heating rate and under 10 bar and 20 bar of 
hydrogen pressure, in the temperature range where the reaction takes place. The values of $p^{*}$ were calculated employing equation (3). From the data included in Figure 6 and equation (2a), it is clear that hydrogen pressure has the effect of decreasing the reaction rate and, therefore, the corresponding DSC curves should shift to higher temperatures, as observed experimentally in Figure 5b. Thus, the hydrogen pressure around the sample has an important influence on the value of the apparent activation energy and the influence of this parameter must be taken into account according to equation (2a) in order to get the real activation energy, E. If the pressure term were neglected, the calculated value of activation energy would be erroneous. This behavior has been suggested for $\mathrm{CaCO}_{3}$ decomposition under $\mathrm{CO}_{2}$ pressure. While calcium carbonate decomposition in vacuum leads to reasonable values of apparent activation energy, decomposition under uncontrolled atmosphere of $\mathrm{CO}_{2}$ gave misleading values of the activation energy and even would lead to an apparent dependence of the activation energy of the reacted fraction $\alpha$ [34].

This is demonstrated if the isoconversional method is applied to the $\mathrm{d} \alpha / \mathrm{dt}-\mathrm{T}$ plots shown in Figure 7, obtained after normalizing the DSC curves presented in Figure 5 as described in the experimental section. Thus, equation $4 \mathrm{~b}$, without accounting for (1$\left.\mathrm{p} / \mathrm{p}^{*}\right)$, was employed for the calculations, and the activation energy values obtained are presented in Table 2. The mean activation energy for $\mathrm{MgH}_{2}$ dehydrogenation under 10 bar is $203 \pm 8 \mathrm{~kJ} \mathrm{~mol}^{-1}$ if calculated without considering the pressure term, which is 92 $\mathrm{kJ} \mathrm{mol}^{-1}$ higher than the value calculated from the experiments carried out under high vacuum. Moreover, a change from $226 \mathrm{~kJ} \mathrm{~mol}^{-1}$ for $\alpha=0.1$ to $195 \mathrm{~kJ} \mathrm{~mol}^{-1}$ for $\alpha=0.6$ is observed, that corresponds to a variation of $31 \mathrm{~kJ} \mathrm{~mol}^{-1}$. This change in activation energy is higher than the expected experimental error. For $\mathrm{MgH}_{2}$ dehydrogenation under 20 bar the mean activation energy is even higher if calculated without considering the pressure term $\left(271 \mathrm{~kJ} \mathrm{~mol}^{-1}\right)$ and a high deviation in the activation energy of $40 \mathrm{~kJ} \mathrm{~mol}^{-1}$ is obtained between the maximum and minimum values of $\alpha$. Therefore, an increase in activation energy is obtained as a function of pressure if the pressure term $\left(1-p / p^{*}\right)$ is not considered in the calculations, and variable activation energies with $\alpha$. 


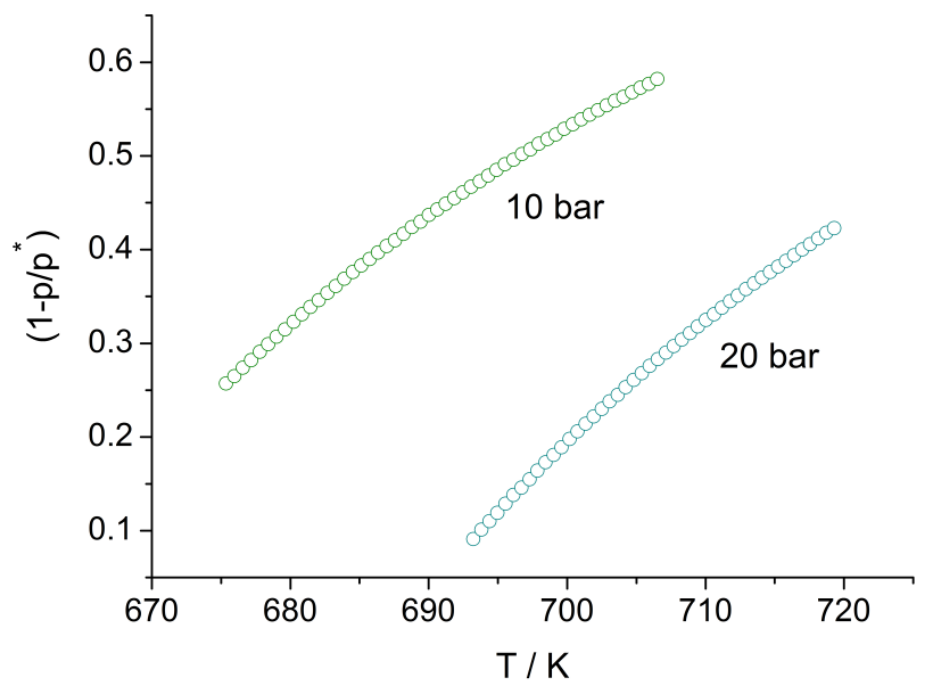

Figure 6. Temperature dependence of the pressure correction term $\left(1-p / p^{*}\right)$ at pressures of 10 bar and 20 bar.
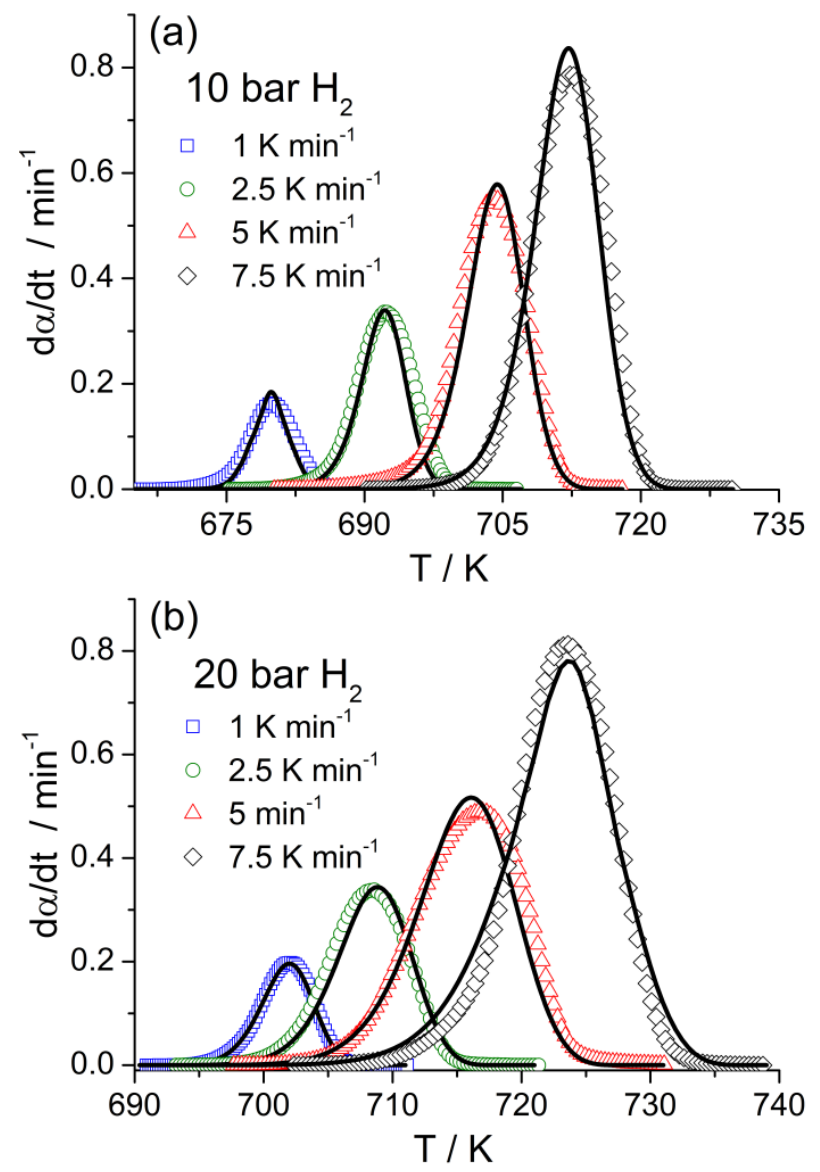

Figure 7. Normalized $\mathrm{d} \alpha / \mathrm{dt}$ plots for the dehydrogenation of magnesium hydride, calculated from the DSC traces shown in figure 5 (dotted lines). a) Hydrogen pressure of 10 bars; b) hydrogen pressure of 20 bars. The curves reconstructed using the kinetic parameters obtained from the kinetic analysis are plotted as solid lines. 
Table 2. Activation energy values as a function of $\alpha$, obtained from the Friedman isoconversional analysis of the experimental curves shown in Figure 7 without accounting for the pressure term $\left(1-\mathrm{p} / \mathrm{p}^{*}\right)$.

\begin{tabular}{|c|c|c|c|c|c|c|c|c|c|}
\hline \multicolumn{10}{|c|}{ dehydrogenation at 10 bar } \\
\hline$\alpha$ & 0.1 & 0.2 & 0.3 & 0.4 & 0.5 & 0.6 & 0.7 & 0.8 & 0.9 \\
\hline $\mathbf{r}$ & 0.996 & 0.996 & 0.997 & 0.998 & 0.997 & 0.998 & 0.998 & 0.998 & 0.999 \\
\hline $\begin{array}{c}E \\
\left(\mathrm{~kJ} \mathrm{~mol}^{-1}\right)\end{array}$ & $226 \pm 9$ & $208 \pm 8$ & $201 \pm 9$ & $197 \pm 7$ & $195 \pm 6$ & $195 \pm 6$ & $197 \pm 5$ & $201 \pm 8$ & $208 \pm 11$ \\
\hline \multicolumn{10}{|c|}{ dehydrogenation at 20 bar } \\
\hline$\alpha$ & 0.1 & 0.2 & 0.3 & 0.4 & 0.5 & 0.6 & 0.7 & 0.8 & 0.9 \\
\hline $\mathbf{r}$ & 0.970 & 0.991 & 0.994 & 0.994 & 0.994 & 0.995 & 0.996 & 0.997 & 0.996 \\
\hline $\begin{array}{c}E \\
\left(\mathrm{~kJ} \mathrm{~mol}^{-1}\right)\end{array}$ & $292 \pm 11$ & $291 \pm 9$ & $283 \pm 7$ & $275 \pm 8$ & $268 \pm 7$ & $263 \pm 6$ & $259 \pm 8$ & $255 \pm 8$ & $252 \pm 10$ \\
\hline
\end{tabular}

Table 3 shows the activation energies obtained by the isoconversional method from the experimental curves included in Figure 7 according to equation (4a), namely considering the pressure term. The activation energies are reasonably constant during the entire processes and, therefore, magnesium hydride dehydrogenation under 10 bar and 20 bar of hydrogen pressure can be described as a single reaction with activation energies averaging $124 \pm 7 \mathrm{~kJ} \mathrm{~mol}^{-1}$ and $127 \pm 4 \mathrm{~kJ} \mathrm{~mol}^{-1}$ respectively. These activation energies match inside the error range attained. Hence, when the pressure term is taken into account, the activation energy obtained is practically constant between 10 and 20 bars and just slightly higher than that calculated for magnesium hydride dehydrogenation under high vacuum.

Table 3. Activation energy values as a function of $\alpha$, obtained from the Friedman isoconversional analysis of the experimental curves shown in Figure 7 accounting for the pressure term $\left(1-\mathrm{p} / \mathrm{p}^{*}\right)$.

\begin{tabular}{|c|c|c|c|c|c|c|c|c|c|}
\hline \multicolumn{10}{|c|}{ dehydrogenation at 10 bar } \\
\hline$\alpha$ & 0.1 & 0.2 & 0.3 & 0.4 & 0.5 & 0.6 & 0.7 & 0.8 & 0.9 \\
\hline $\mathbf{r}$ & 0.995 & 0.999 & 0.999 & 0.999 & 0.999 & 0.999 & 0.999 & 0.999 & 0.997 \\
\hline $\begin{array}{c}E \\
\left(\mathrm{~kJ} \mathrm{~mol}^{-1}\right)\end{array}$ & $131 \pm 11$ & $123 \pm 6$ & $119 \pm 5$ & $118 \pm 7$ & $118 \pm 5$ & $120 \pm 5$ & $125 \pm 4$ & $127 \pm 7$ & $135 \pm 12$ \\
\hline \multicolumn{10}{|c|}{ dehydrogenation at 20 bar } \\
\hline$\alpha$ & 0.1 & 0.2 & 0.3 & 0.4 & 0.5 & 0.6 & 0.7 & 0.8 & 0.9 \\
\hline $\mathbf{r}$ & 0.968 & 0.991 & 0.993 & 0.994 & 0.994 & 0.995 & 0.996 & 0.997 & 0.996 \\
\hline $\begin{array}{c}E \\
\left(\mathrm{~kJ} \mathrm{~mol}^{-1}\right)\end{array}$ & $121 \pm 7$ & $128 \pm 4$ & $129 \pm 5$ & $127 \pm 3$ & $126 \pm 3$ & $125 \pm 3$ & $126 \pm 4$ & $128 \pm 5$ & $131 \pm 8$ \\
\hline
\end{tabular}


The combined kinetic analysis, described in Section 3.2, was applied to the curves registered under 10 bar and 20 bar of $\mathrm{H}_{2}$ separately. Figure 8 shows the plots of the left hand side of equation $5 \mathrm{a}$, using each set of experimental data, versus the reverse of temperature. The entire conversion range can be reasonably fitted to the model for both experiments under 10 bar and 20 bar. The values obtained for $\mathrm{n}$ and $\mathrm{m}$ from the optimization procedure are $n=0.881$ and $m=0.864$ at 10 bar and $n=0.83$ and $m=$ 0.745 at 20 bar of hydrogen pressure. The slopes of the plots lead to activation energy values of $124 \pm 2 \mathrm{~kJ} \mathrm{~mol}^{-1}$ and $121 \pm 4 \mathrm{~kJ} \mathrm{~mol}^{-1}$ for $10 \mathrm{bar}$ and $20 \mathrm{bar}$, respectively, while the intercepts yield to values of cA of $5.4( \pm 1.5) \times 10^{9} \mathrm{~min}^{-1}$ and $2.9( \pm 0.8) \times 10^{9}$ $\min ^{-1}$. The value of $\mathrm{c}$ cannot be discriminated but a combined $\mathrm{cA}$ is obtained instead. The activation energy values are in good agreement with those calculated by the isoconversional analysis (Table 3), which demonstrates the reliability of the values obtained.

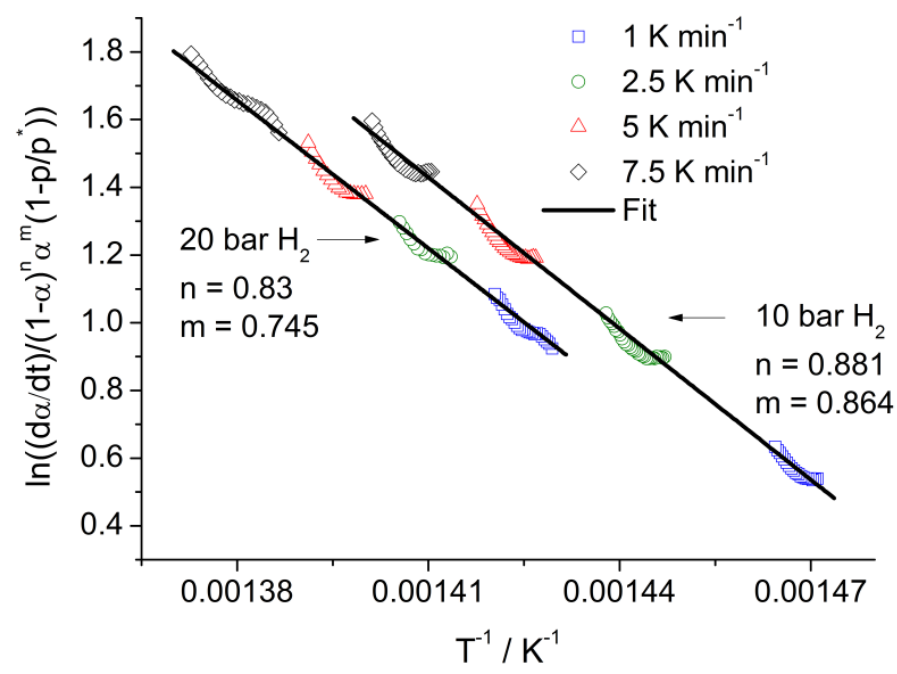

Figure 8. Combined kinetic analysis plots of the experimental curves presented in Figure 7, recorded in 10 bar and 20 bar of hydrogen pressure.

Comparison with the theoretical kinetic functions is needed to determine the kinetic model followed by the reaction. In figure 9 , the conversion functions estimated by the combined kinetic analysis are plotted together with the most commonly used kinetic models, such as nucleation and growth, first order and diffusion. The comparison evidences that the calculated $\mathrm{f}(\alpha)$ functions obtained for $\mathrm{MgH} 2$ dehydrogenation under 10 bar and 20 bar match the master plot corresponding to the A3 Avrami-Erofeev 
kinetic model. It is noteworthy remark that these results have been obtained without any previous assumption about the kinetic models or the activation energies. From a physical point of view, this model implies the instant formation of tridimensional nuclei followed by the growth of these nuclei [61].

In the same way as was done in the previous section for $\mathrm{MgH}_{2}$ dehydrogenation under high vacuum, the kinetic parameters calculated by the kinetic analysis were used for reconstructing the experimental curves numerically, integrating the general kinetic equation and considering the heating conditions used in the different experiments. Then, the curves were differentiated with respect to time and the resulting $\mathrm{d} \alpha / \mathrm{dt}$ curves are included in Figure 7. The kinetic parameters are validated by the close match between the simulated $\mathrm{d} \alpha / \mathrm{dt}-\mathrm{T}$ curves and the experimental ones.
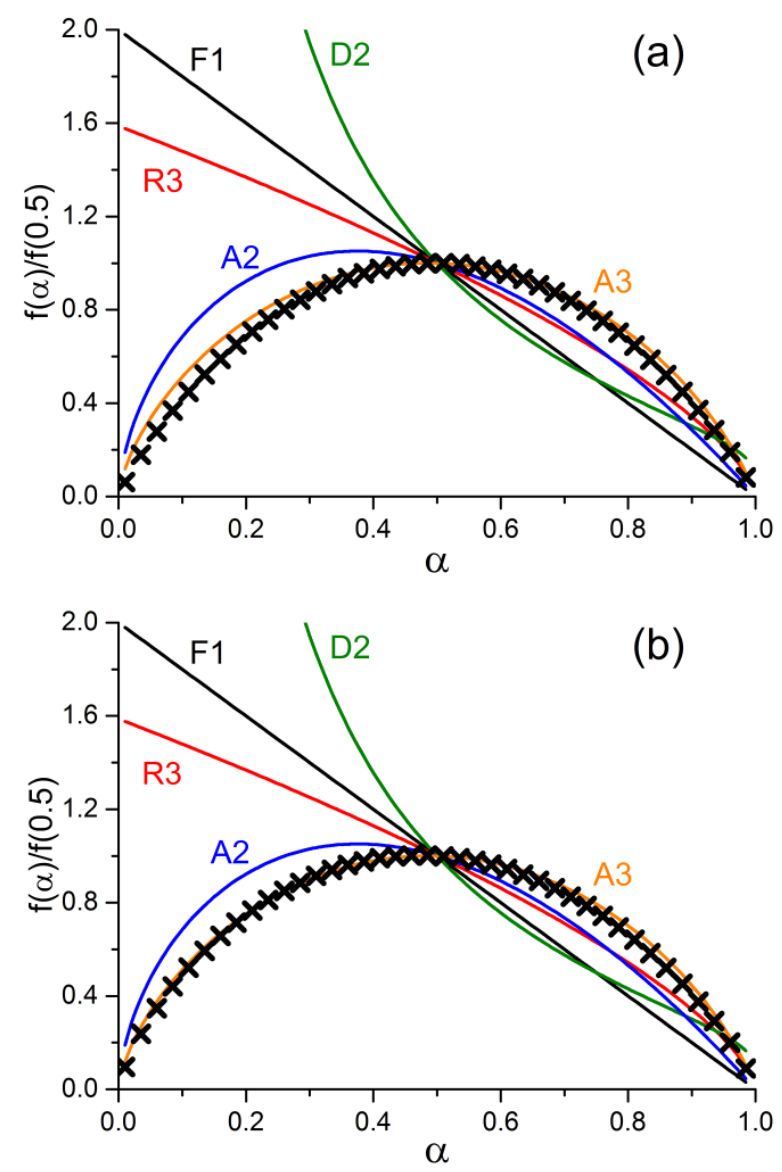

Figure 9. Comparison of the $f(\alpha)$ functions (lines) normalized at $\alpha=0.5$ corresponding to some of the ideal kinetic models with the $\mathrm{f}(\alpha)$ functions resulting from the combined analysis of $\mathrm{MgH}_{2}$ dehydrogenation in (a) 10 bar of hydrogen pressure and (b) 20 bar of hydrogen pressure. 
The dehydrogenation mechanism is modified by the experimental conditions in which the experiments are performed. Thus, a change from first-order driven process (equivalent to A1) in high vacuum to an A3 nucleation mechanism in hydrogen pressure is observed. This change of mechanism would be explained taking into account the large increase of the decomposition temperature imposed by the equilibrium displacement under hydrogen pressure. This noticeably increase of the decomposition temperature of the hydride would promote the growth of the potential nucleus forming sites, until collapsing in nuclei of tridimensional shape. Thus, once that the equilibrium temperature for the dehydrogenation of $\mathrm{MgH}_{2}$ under a given hydrogen pressure is overpassed, the dehydrogenation reaction takes place through the tridimensional growth of the nuclei previously formed.

On the other hand, it is noteworthy to point out that the difference between the activation energies obtained under high vacuum and under hydrogen pressure is only about a $12 \%$. The fact that the activation energy of the forward reaction obtained under hydrogen pressure thoroughly agree with the value of $127 \mathrm{~kJ} \mathrm{~mol}^{-1}$ reported for the $\mathrm{Mg}$ $\mathrm{H}$ bond energy suggests that the breaking of this bond is the rate limiting step of magnesium hydride thermal dehydrogenation [62]. The extra energy stored as lattice defects on the starting sample would account for the slightly lower activation energy obtained for the dehydrogenation of $\mathrm{MgH}_{2}$ under high vacuum.

Finally, the fact that activation energies closed to $120 \mathrm{~kJ} \mathrm{~mol}^{-1}$ were determined for the dehydrogenation kinetics under isothermal conditions using as raw materials $\mathrm{MgH}_{2}$ samples supplied by different manufacturers, as shown in a recent review [3], would suggest that the $\mathrm{Mg}-\mathrm{H}$ bond-breaking could be the rate limiting step of the reaction as we have proposed. This would be true in spite that different kinetic models were assumed for determining the activation energies collected in the Varin et al. review [3]. It has been demonstrated in a recent paper that the same activation energy is obtained from a set of isothermal data, whatever would be the kinetic model previously assumed, which is not true if kinetic data obtained from rising temperature experiments are concerned [63].

\section{Conclusions}

Kinetics of $\mathrm{MgH}_{2}$ dehydrogenation has been studied in three different experimental conditions. The kinetic analysis has been performed using model-free isoconversional 
analysis that provides the value of the activation energy as a function of the reaction fraction, and the combined kinetic analysis procedure that allows obtaining all the kinetic parameters avoiding any kind of assumption about the kinetic model obeyed by the reaction, and therefore the risk of model-fitting the experimental data with an erroneous kinetic model. The reliability of the calculated kinetic parameters has been tested by comparing simulated and experimental curves.

Interestingly, results obtained show that dehydrogenation mechanism of $\mathrm{MgH}_{2}$ depends on the experimental conditions used to carry out the reaction. Thus, if the reaction is performed under high vacuum, it follows a first order kinetics, equivalent to an AvramiErofeev kinetic model with an Avrami coefficient equal to 1, while a tridimensional growth of nuclei previously formed (A3) is followed when the experiment is carried out under 10 bar or 20 bar of hydrogen pressure. A unified theory that explains this behavior is given. The activation energy of the reaction is less influenced by the experimental conditions, and the values obtained agree with the value reported for $\mathrm{Mg}$ $\mathrm{H}$ bond energy, which suggests that the breaking of this bond could be the rate limiting step of the $\mathrm{MgH}_{2}$ thermal dehydrogenation.

\section{Acknowledgements}

It is acknowledged the support from projects CTQ2014-52763-C2-1-R (MINECOFEDER), TEP-7858 (Junta Andalucía-FEDER) and TEP-1900 (Junta AndalucíaFEDER). The authors also thank VPPI-US for the AP current contract. Additionally, one of the authors (PESJ) is supported by a Marie Curie-Junta de Andalucía Talentia grant.

\section{References}

[1] Grochala, W., Edwards, P. P., Thermal decomposition of the non-interstitial hydrides for the storage and production of hydrogen, Chem. Rev. 104 (2004) 1283-1315.

[2] Imamura, H., Masanari, K., Kusuhara, M., Katsumoto, H., Sumi, T., Sakata, Y., High hydrogen storage capacity of nanosized magnesium synthesized by high energy ballmilling, J. Alloy. Compd. 386 (2005) 211-216.

[3] Varin, R. A. C., Tomasz; Wronski, Zbigniew S., Nanomaterials for solid state hydrogen storage, Springer Science-Business Media, New York, 2009.

[4] Zaluska, A., Zaluski, L., Strom-Olsen, J. O., Nanocrystalline magnesium for hydrogen storage, J. Alloy. Compd. 288 (1999) 217-225. 
[5] Zaluska, A., Zaluski, L., Strom-Olsen, J. O., Structure, catalysis and atomic reactions on the nano-scale: A systematic approach to metal hydrides for hydrogen storage, Appl. Phys. A-Mater. 72 (2001) 157-165.

[6] Bogdanovic, B., Hartwig, T. H., Spliethoff, B., The development, testing and optimization of energy-storage materials based on the $\mathrm{MgH}_{2}-\mathrm{Mg}$ system, Int. J. Hydrogen Energ. 18 (1993) 575-589.

[7] Bogdanovic, B., Ritter, A., Spliethoff, B., Active $\mathrm{MgH}_{2}-\mathrm{Mg}$ systems for reversible chemical energy-storage, Angew. Chem. Int. Edit. 29 (1990) 223-234.

[8] Bogdanovic, B., Ritter, A., Spliethoff, B., A process steam-generator based on the hightemperature magnesium hydride magnesium heat-storage system, Int. J. Hydrogen Energ. 20 (1995) 811-822.

[9] Reiser, A., Bogdanovic, B., Schlichte, K., The application of Mg-based metal-hydrides as heat energy storage systems, Int. J. Hydrogen Energ. 25 (2000) 425-430.

[10] Wierse, M., Werner, R., Groll, M., Magnesium hydride for thermal-energy storage in a small-scale solar thermal power-station, J. Less-Common Met. 172 (1991) 1111-1121.

[11] Huot, J., Liang, G., Boily, S., Van Neste, A., Schulz, R., Structural study and hydrogen sorption kinetics of ball-milled magnesium hydride, J. Alloy. Compd. 293 (1999) 495500.

[12] Barkhordarian, G., Klassen, T., Bormann, R., Fast hydrogen sorption kinetics of nanocrystalline mg using $\mathrm{Nb}_{2} \mathrm{O}_{5}$ as catalyst, Scripta Mater. 49 (2003) 213-217.

[13] Kodera, Y., Yamasaki, N., Yamamoto, T., Kawasaki, T., Ohyanagi, M., Munir, Z. A., Hydrogen storage $\mathrm{Mg}_{2} \mathrm{Ni}$ alloy produced by induction field activated combustion synthesis, J. Alloy. Compd. 446 (2007) 138-141.

[14] Liu, X., Zhu, Y., Li, L., Hydriding and dehydriding properties of nanostructured $\mathrm{Mg}_{2} \mathrm{Ni}$ alloy prepared by the process of hydriding combustion synthesis and subsequent mechanical grinding, J. Alloy. Compd. 425 (2006) 235-238.

[15] Shang, C. X., Guo, Z. X., Structural and desorption characterisations of milled $\left(\mathrm{MgH}_{2}+\mathrm{Y}\right.$, Ce) powder mixtures for hydrogen storage, Int. J. Hydrogen Energ. 32 (2007) 29202925.

[16] Varin, R. A., Li, S., Wronski, Z., Morozova, O., Khomenko, T., The effect of sequential and continuous high-energy impact mode on the mechano-chemical synthesis of nanostructured complex hydride $\mathrm{Mg}_{2} \mathrm{FeH}_{6}$ J. Alloy. Compd. 390 (2005) 282-296.

[17] Bohmhammel, K., Christ, B., Wolf, G., Kinetic investigations on the basis of isothermal DSC measurements of hydrogenation and dehydrogenation of magnesium hydride, Thermochim. Acta 310 (1998) 167-171.

[18] Fernandez, J. F., Sanchez, C. R., Rate determining step in the absorption and desorption of hydrogen by magnesium, J. Alloy. Compd. 340 (2002) 189-198.

[19] Cermak, J., Kral, L., Hydrogen diffusion in Mg-H and Mg-Ni-H alloys, Acta Mater. 56 (2008) 2677-2686.

[20] Yao, X., Zhu, Z. H., Cheng, H. M., Lu, G. Q., Hydrogen diffusion and effect of grain size on hydrogenation kinetics in magnesium hydrides, J. Mater. Res. 23 (2008) 336-340.

[21] Campostrini, R., Abdellatief, M., Leoni, M., Scardi, P., Activation energy in the thermal decomposition of $\mathrm{MgH}_{2}$ powders by coupled TG-MS measurements, J. Therm. Anal. Calorim. 116 (2014) 225-240.

[22] Evard, E., Gabis, I., Yartys, V. A., Kinetics of hydrogen evolution from $\mathrm{MgH}_{2}$ : Experimental studies, mechanism and modelling, Int. J. Hydrogen Energ. 35 (2010) 9060-9069.

[23] Gerasimov, K. B., Ivanov, E. Y., The mechanism and kinetics of formation and decomposition of magnesium hydride, Mater. Lett. 3 (1985) 497-499.

[24] Jensen, T. R., Andreasen, A., Vegge, T., Andreasen, J. W., Stahl, K., Pedersen, A. S., Nielsen, M. M., Molenbroek, A. M., Besenbacher, F., Dehydrogenation kinetics of pure 
and nickel-doped magnesium hydride investigated by in situ time-resolved powder XRay diffraction, Int. J. Hydrogen Energ. 31 (2006) 2052-2062.

[25] Liang, G., Huot, J., Boily, S., Schulz, R., Hydrogen desorption kinetics of a mechanically milled $\mathrm{MgH}_{2}$-5at.\%V nanocomposite, J. Alloy. Compd. 305 (2000) 239-245.

[26] Ron, M., The normalized pressure dependence method for the evaluation of kinetic rates of metal hydride formation/decomposition, J. Alloy. Compd. 283 (1999) 178-191.

[27] Song, M. Y., Manaud, J. P., Darriet, B., Dehydriding kinetics of a mechanically alloyed mixture Mg-10wt.\%Ni, J. Alloy. Compd. 282 (1999) 243-247.

[28] Stander, C. M., Kinetics of decomposition of magnesium hydride, J. Inorg. Nucl. Chem. 39 (1977) 221-223.

[29] Brown, M. E., Steps in a minefield - Some kinetic aspects of thermal analysis, J. Therm. Anal. 49 (1997) 17-32.

[30] Reading, M., Dollimore, D., Whitehead, R., The measurement of meaningful kineticparameters for solid-state decomposition reactions, J. Therm. Anal. 37 (1991) 21652188.

[31] Sanchez-Jimenez, P. E., Perez-Maqueda, L. A., Perejon, A., Criado, J. M., Clarifications regarding the use of model-fitting methods of kinetic analysis for determining the activation energy from a single non-isothermal curve, Chem. Cent. J. 7 (2013) 25.

[32] Vyazovkin, S., Burnham, A. K., Criado, J. M., Pérez-Maqueda, L. A., Popescu, C., Sbirrazzuoli, N., ICTAC kinetics committee recommendations for performing kinetic computations on thermal analysis data, Thermochim. Acta 520 (2011) 1-19.

[33] Vyazovkin, S. V., Lesnikovich, A. I., On the methods of solving the inverse problem of solid-phase reaction-kinetics. 1. Methods based on discrimination, J. Therm. Anal. 35 (1989) 2169-2188.

[34] Criado, J. M., Gonzalez, M., Malek, J., Ortega, A., The effect of the $\mathrm{CO}_{2}$ pressure on the thermal-decomposition kinetics of calcium-carbonate, Thermochim. Acta 254 (1995) 121-127.

[35] Tan, G., Wang, Q., Zheng, H., Zhao, W., Zhang, S., Liu, Z., Concept of variable activation energy and its validity in nonisothermal kinetics, J. Phys. Chem. A 115 (2011) 55175524.

[36] Barret, P., C. R. Acad. Sci., Ser. C, 266 (1968) 856.

[37] Searcy, A. W., Beruto, D., Kinetics of endothermic decomposition reactions. 2. Effects of the solid and gaseous products, J. Phys. Chem. 82 (1978) 163-167.

[38] Criado, J. M., Sanchez-Jimenez, P. E., Perez-Maqueda, L. A., Critical study of the isoconversional methods of kinetic analysis, J. Therm. Anal. Calorim. 92 (2008) 199203.

[39] Friedman, H. L., Kinetics of thermal degradation of char-forming plastics from thermogravimetry. Application to phenolic plastic, J. Polym. Sci. Pol. Sym. (1964) 183195.

[40] Perez-Maqueda, L. A., Criado, J. M., Sanchez-Jimenez, P. E., Combined kinetic analysis of solid-state reactions: A powerful tool for the simultaneous determination of kinetic parameters and the kinetic model without previous assumptions on the reaction mechanism, J. Phys. Chem. A 110 (2006) 12456-12462.

[41] Sanchez-Jimenez, P. E., Perez-Maqueda, L. A., Perejon, A., Criado, J. M., Combined kinetic analysis of thermal degradation of polymeric materials under any thermal pathway, Polym. Degrad. Stabil. 94 (2009) 2079-2085.

[42] Koga, N., Criado, J. M., Influence of the particle size distribution on the CRTA curves for the solid-state reactions of interface shrinkage, J. Therm. Anal. 49 (1997) 1477-1484.

[43] Koga, N., Criado, J. M., Kinetic analyses of solid-state reactions with a particle-size distribution, J. Am. Ceram. Soc. 81 (1998) 2901-2909. 
[44] Grau-Crespo, R., Smith, K. C., Fisher, T. S., de Leeuw, N. H., Waghmare, U. V., Thermodynamics of hydrogen vacancies in $\mathrm{MgH}_{2}$ from first-principles calculations and grand-canonical statistical mechanics, Phys. Rev. B 80 (2009) 6174117.

[45] Schimmel, H. G., Huot, J., Chapon, L. C., Tichelaar, F. D., Mulder, F. M., Hydrogen cycling of niobium and vanadium catalyzed nanostructured magnesium, J. Am. Chem. Soc. 127 (2005) 14348-14354.

[46] Gerard, N., Ono, S., Hydrogen in Intermetallic Compounds II, Springer Verlag, 1992.

[47] Sastri, M. V. C., Viswanathan, B., Srinivasa Murthy, S., Metal Hydrides: Fundamentals and Applications, Springer-Verlag, 1998.

[48] Zhou, C. S., Fang, Z. G. Z., Lu, J., Zhang, X. Y., Thermodynamic and kinetic destabilization of magnesium hydride using Mg-In solid solution alloys, J. Am. Chem. Soc. 135 (2013) 10982-10985.

[49] Zhou, C. S., Fang, Z. Z., Lu, J., Luo, X. Y., Ren, C., Fan, P., Ren, Y., Zhang, X. Y., Thermodynamic destabilization of magnesium hydride using Mg-based solid solution alloys, J. Phys. Chem. C 118 (2014) 11526-11535.

[50] Gotor, F. J., Criado, J. M., Malek, J., Koga, N., Kinetic analysis of solid-state reactions: The universality of master plots for analyzing isothermal and nonisothermal experiments, J. Phys. Chem. A 104 (2000) 10777-10782.

[51] Hulbert, S. F., Models of solid state reactions in powder compacts. A review, J. Brit. Ceram. Soc. 6 (1969) 11-20.

[52] Perez-Maqueda, L. A., Criado, J. M., Malek, J., Combined kinetic analysis for crystallization kinetics of non-crystalline solids, J. Non-Cryst. Solids 320 (2003) 84-91.

[53] Pijolat, M., Soustelle, M., Experimental tests to validate the rate-limiting step assumption used in the kinetic analysis of solid-state reactions, Thermochim. Acta 478 (2008) 34-40.

[54] Young, D. A., Decomposition of solids, Pergamon Press, Oxford, 1966.

[55] Criado, J. M., Morales, J., Defects of thermogravimetric analysis for discerning between first order reactions and those taking place through the Avrami-Erofeev's mechanism, Thermochim. Acta 16 (1976) 382-387.

[56] Criado, J. M., Morales, J., Thermal decomposition reactions of solids controlled by diffusion and phase-boundary processes: possible misinterpretation of the mechanism from thermogravimetric data, Thermochim. Acta 19 (1977) 305-317.

[57] Aguey-Zinsou, K. F., Ares-Fernandez, J. R., Hydrogen in magnesium: New perspectives toward functional stores, Energ. Environ. Sci. 3 (2010) 526-543.

[58] Bogdanovic, B., Bohmhammel, K., Christ, B., Reiser, A., Schlichte, K., Vehlen, R., Wolf, U., Thermodynamic investigation of the magnesium-hydrogen system, J. Alloy. Compd. 282 (1999) 84-92.

[59] Bohmhammel, K., Wolf, U., Wolf, G., Konigsberger, E., Thermodynamic optimization of the system magnesium-hydrogen, Thermochim. Acta 337 (1999) 195-199.

[60] Broom, D. P., Hydrogen storage materials. The characterisation of their storage properties, Springer London, London, 2011.

[61] Khawam, A., Flanagan, D. R., Solid-state kinetic models: Basics and mathematical fundamentals, J. Phys. Chem. B 110 (2006) 17315-17328.

[62] Luo, Y. R., in CRC Handbook of Physical and Chemistry, $96^{\text {th }}$ Edition, Haynes W.M., CRC Press/Taylor and Francys, Boca Raton, USA, 2014, pp. 9-65.

[63] Sanchez-Jimenez, P. E., Perejon, A., Perez-Maqueda, L. A., Criado, J. M., New insights on the kinetic analysis of isothermal data: The independence of the activation energy from the assumed kinetic model, Energ. Fuel. 29 (2015) 392-397. 